\title{
LOCAL REGULARITY FOR FRACTIONAL HEAT EQUATIONS
}

\author{
UMBERTO BICCARI, MAHAMADI WARMA, AND ENRIQUE ZUAZUA
}

\begin{abstract}
We prove the maximal local regularity of weak solutions to the parabolic problem associated with the fractional Laplacian with homogeneous Dirichlet boundary conditions on an arbitrary bounded open set $\Omega \subset \mathbb{R}^{N}$. Proofs combine classical abstract regularity results for parabolic equations with some new local regularity results for the associated elliptic problems.
\end{abstract}

\section{Dedicated to Enrique Fernández-Cara on his 60th birthday.}

\section{INTRODUCTION}

The aim of the present paper is to study the local regularity of weak solutions to the following parabolic problem

$$
\begin{cases}u_{t}+(-\Delta)^{s} u=f & \text { in } \Omega \times(0, T)=: \Omega_{T}, \\ u \equiv 0 & \text { on }\left(\mathbb{R}^{N} \backslash \Omega\right) \times(0, T), \\ u(\cdot, 0) \equiv 0 & \text { in } \Omega,\end{cases}
$$

where $\Omega \subset \mathbb{R}^{N}$ is an arbitrary bounded open set, $f$ is a given distribution and, for all $s \in(0,1),(-\Delta)^{s}$ denotes the fractional Laplace operator, which is defined as the following singular integral

$$
(-\Delta)^{s} u(x):=C_{N, s} \text { P.V. } \int_{\mathbb{R}^{N}} \frac{u(x)-u(y)}{|x-y|^{N+2 s}} d y, \quad x \in \mathbb{R}^{N} .
$$

In (1.2), $C_{N, s}$ is a normalization constant given by

$$
C_{N, s}:=\frac{s 2^{2 s} \Gamma\left(\frac{2 s+N}{2}\right)}{\pi^{\frac{N}{2}} \Gamma(1-s)},
$$

$\Gamma$ being the usual Gamma function.

We are interested in analyzing the local regularity for solutions to the parabolic problem (1.1).

We first introduce the functional setting. Given $\Omega \subset \mathbb{R}^{N}$, an arbitrary open set, for $p \in(1, \infty)$ and $s \in(0,1)$, we denote by

$$
W^{s, p}(\Omega):=\left\{u \in L^{p}(\Omega): \int_{\Omega} \int_{\Omega} \frac{|u(x)-u(y)|^{p}}{|x-y|^{N+p s}} d x d y<\infty\right\},
$$

the fractional order Sobolev space endowed with the norm

$$
\|u\|_{W^{s, p}(\Omega)}:=\left(\int_{\Omega}|u|^{p} d x+\int_{\Omega} \int_{\Omega} \frac{|u(x)-u(y)|^{p}}{|x-y|^{N+p s}} d x d y\right)^{\frac{1}{p}} .
$$

2010 Mathematics Subject Classification. 35B65, 35R11, 35S05.

Key words and phrases. Fractional Laplacian, Heat equation, Dirichlet boundary condition, weak solutions, local regularity.

The work of Umberto Biccari was partially supported by the Advanced Grant DYCON (Dynamic Control) of the European Research Council Executive Agency, by the MTM2014-52347 Grant of the MINECO (Spain) and by the Air Force Office of Scientific Research under the Award No: FA9550-15-1-0027. The work of Mahamadi Warma was partially supported by the Air Force Office of Scientific Research under the Award No: FA9550-15-1-0027. The work of Enrique Zuazua was partially supported by the Advanced Grant DYCON (Dynamic Control) of the European Research Council Executive Agency, FA9550-15-1-0027 of AFOSR, FA9550-14-1-0214 of the EOARD-AFOSR, the MTM2014-52347 Grant of the MINECO (Spain) and ICON of the French ANR. 
We let

$$
W_{0}^{s, p}(\bar{\Omega}):=\left\{u \in W^{s, p}\left(\mathbb{R}^{N}\right): u=0 \text { on } \mathbb{R}^{N} \backslash \Omega\right\},
$$

and we shall denote by $W^{-s, 2}(\bar{\Omega})$ the dual of the Hilbert space $W_{0}^{s, 2}(\bar{\Omega})$, that is, $W^{-s, 2}(\bar{\Omega}):=\left(W_{0}^{s, 2}(\bar{\Omega})\right)^{\star}$. The following continuous embeddings hold

$$
W_{0}^{s, 2}(\bar{\Omega}) \hookrightarrow L^{2}(\Omega) \hookrightarrow W^{-s, 2}(\bar{\Omega}) .
$$

Next, if $s>1$ is not an integer, we write $s=m+\sigma$ where $m$ is an integer and $0<\sigma<1$. In this case

$$
W^{s, p}(\Omega):=\left\{u \in W^{m, p}(\Omega): D^{\alpha} u \in W^{\sigma, p}(\Omega) \text { for any } \alpha \text { such that }|\alpha|=m\right\} \text {. }
$$

Then $W^{s, p}(\Omega)$ is a Banach space with respect to the norm

$$
\|u\|_{W^{s, p}(\Omega)}:=\left(\|u\|_{W^{m, p}(\Omega)}^{p}+\sum_{|\alpha|=m}\left\|D^{\alpha} u\right\|_{W^{\sigma, p}(\Omega)}^{p}\right)^{\frac{1}{p}} .
$$

If $s=m$ is an integer, then $W^{s, p}(\Omega)$ coincides with the classical integral order Sobolev space $W^{m, p}(\Omega)$.

We also recall the following definition of the Besov space $B_{p, q}^{s}$, according to [16. Chapter V, Section 5.1, Formula (60)]:

$$
B_{p, q}^{s}\left(\mathbb{R}^{N}\right):=\left\{u \in L^{p}\left(\mathbb{R}^{N}\right):\left(\int_{\mathbb{R}^{N}} \frac{\|u(x+y)-u(y)\|_{L^{p}\left(\mathbb{R}^{N}\right)}^{q}}{|y|^{N+q s}} d y\right)^{\frac{1}{q}}<\infty\right\}, \quad 1 \leq p, q \leq \infty, \quad 0<s<1 .
$$

Notice that, when $p=q$, we have $B_{p, p}^{s}\left(\mathbb{R}^{N}\right)=W^{s, p}\left(\mathbb{R}^{N}\right)$. Finally, we recall the definition of the following potential space

$$
\mathscr{L}_{2 s}^{p}\left(\mathbb{R}^{N}\right):=\left\{u \in L^{p}\left(\mathbb{R}^{N}\right):(-\Delta)^{s} u \in L^{p}\left(\mathbb{R}^{N}\right)\right\}, \quad 1 \leq p \leq \infty, \quad s \geq 0
$$

introduced, for example, in [16, Chapter V, Section 3.3, Formula (38)]. Note that this same space is sometimes denoted as $H_{p}^{s}\left(\mathbb{R}^{N}\right.$ ) (see, e.g., [18, Section 1.3.2]). Here we adopt the notation $\mathscr{L}_{2 s}^{p}\left(\mathbb{R}^{N}\right)$.

Let us now introduce the notion of solution that we shall consider. Following [12], we first consider weak solutions of (1.1) defined as follows.

Definition 1.1. Let $f \in L^{2}\left((0, T) ; W^{-s, 2}(\bar{\Omega})\right)$. We say that $u \in L^{2}\left((0, T) ; W_{0}^{s, 2}(\bar{\Omega})\right) \cap C\left([0, T] ; L^{2}(\Omega)\right)$ with $u_{t} \in L^{2}\left((0, T) ; W^{-s, 2}(\bar{\Omega})\right)$ is a finite energy solution to the parabolic problem (1.1), if the identity

$$
\begin{aligned}
& \int_{0}^{T} \int_{\Omega} u_{t} w d x d t+\frac{C_{N, s}}{2} \int_{0}^{T} \int_{\mathbb{R}^{N}} \int_{\mathbb{R}^{N}} \frac{(u(x)-u(y))(w(x)-w(y))}{|x-y|^{N+2 s}} d x d y d t \\
= & \int_{0}^{T}\langle f, v\rangle_{W^{-s, 2}(\bar{\Omega}), W_{0}^{s, 2}(\bar{\Omega})} d t,
\end{aligned}
$$

holds, for any $w \in L^{2}\left((0, T) ; W_{0}^{s, 2}(\bar{\Omega})\right)$, where $\langle\cdot, \cdot\rangle_{W^{-s, 2}(\bar{\Omega}), W_{0}^{s, 2}(\bar{\Omega})}$ denotes the duality pairing between $W^{-s, 2}(\bar{\Omega})$ and $W_{0}^{s, 2}(\bar{\Omega})$.

Remark 1.2. We observe the following facts.

(a) According to [12, Theorem 10], if $u \in L^{2}\left((0, T) ; W_{0}^{s, 2}(\bar{\Omega})\right)$ and $u_{t} \in L^{2}\left((0, T) ; W^{-s, 2}(\bar{\Omega})\right)$, then $u \in C\left([0, T] ; L^{2}(\Omega)\right)$. Thus the identity $u(\cdot, 0)=0$ makes sense in $L^{2}(\Omega)$.

(b) When considering right hand side terms $f \in L^{p}\left((0, T) ; L^{p}(\Omega)\right)=L^{p}(\Omega \times(0, T))$ with $p \geq 2$, since we have the continuous embedding $L^{p}(\Omega \times(0, T)) \hookrightarrow L^{2}\left((0, T) ; W^{-s, 2}(\bar{\Omega})\right)$, this notion of weak finite energy solution suffices.

(c) When $f \in L^{p}(\Omega \times(0, T))$ with $1 \leq p<2$, the regularity of the right hand side term does not suffice to define weak finite energy solutions as above. We shall rather consider those defined by duality or transposition. 
Duality or transposition solutions of (1.1) are given by duality with respect to the following class of test functions

$$
\mathcal{P}\left(\Omega_{T}\right)=\left\{\phi(\cdot, t) \in C^{1}\left((0, T), C_{0}^{\beta}(\Omega)\right): \phi \text { is a solution to Problem }(\mathrm{P})\right\},
$$

where

$$
(P)= \begin{cases}-\phi_{t}+(-\Delta)^{s} \phi=\psi & \text { in } \Omega \times(0, T)=: \Omega_{T}, \\ \phi \equiv 0 & \text { on }\left(\mathbb{R}^{N} \backslash \Omega\right) \times(0, T), \\ \phi(\cdot, T) \equiv 0 & \text { in } \Omega\end{cases}
$$

for $\psi \in C_{0}^{\infty}\left(\Omega_{T}\right)$.

Definition 1.3. Let $f \in L^{1}(\Omega \times(0, T))$. We say that $u \in C\left([0, T] ; L^{1}(\Omega)\right)$ is a weak duality or transposition solution to the parabolic problem (1.1), if the identity

$$
\int_{0}^{T} \int_{\Omega} u \psi d x d t=\int_{0}^{T} \int_{\Omega} f \phi d x d t
$$

holds, for any $\phi \in \mathcal{P}\left(\Omega_{T}\right)$ and $\psi \in C_{0}^{\infty}\left(\Omega_{T}\right)$.

Remark 1.4. The existence and uniqueness of finite energy weak solutions or the duality/transposition ones (depending on the regularity imposed on the right hand side term $f$ ) to problem (1.1) is guaranteed by [12. Theorem 26] and [12, Theorem 28], respectively. If $f \in L^{p}(\Omega \times(0, T))$, with $p \geq 2$, finite energy solutions of (1.1) will be considered while, if $1<p<2$, solutions will be understood in the sense of duality/transposition. In both cases we shall refer to them as weak solutions.

Our first regularity result concerns the case $p=2$. It reads as follows:

Theorem 1.5. Assume $f \in L^{2}(\Omega \times(0, T))$ and let $u \in L^{2}\left((0, T) ; W_{0}^{s, 2}(\bar{\Omega})\right) \cap C\left([0, T] ; L^{2}(\Omega)\right)$ with $u_{t} \in L^{2}\left((0, T) ; W^{-s, 2}(\bar{\Omega})\right)$ be the unique finite energy solution of system (1.1). Then

$$
u \in L^{2}\left((0, T) ; W_{\mathrm{loc}}^{2 s, 2}(\Omega)\right) \cap L^{\infty}\left((0, T) ; W_{0}^{s, 2}(\bar{\Omega})\right) \text { and } u_{t} \in L^{2}(\Omega \times(0, T)) .
$$

Theorem 1.5 can be extended to the $L^{p}$-setting as follows.

Theorem 1.6. Let $1<p<\infty$ and $f \in L^{p}(\Omega \times(0, T))$. Then, problem (1.1) has a unique weak solution $u \in C\left([0, T] ; L^{p}(\Omega)\right)$ such that $u \in L^{p}\left((0, T) ; \mathscr{L}_{2 s, \mathrm{loc}}^{p}(\Omega)\right)$ and $u_{t} \in L^{p}(\Omega \times(0, T))$. As a consequence we have the following result.

(a) If $1<p<2$ and $s \neq 1 / 2$, then $u \in L^{p}\left((0, T) ; B_{p, 2, \text { loc }}^{2 s}(\Omega)\right)$.

(b) If $1<p<2$ and $s=1 / 2$, then $u \in L^{p}\left((0, T) ; W_{\mathrm{loc}}^{2 s, p}(\Omega)\right)=L^{p}\left((0, T) ; W_{\mathrm{loc}}^{1, p}(\Omega)\right)$.

(c) If $2 \leq p<\infty$, then $u \in L^{p}\left((0, T) ; W_{\mathrm{loc}}^{2 s, p}(\Omega)\right)$.

In Theorem 1.6, with $\mathscr{L}_{2 s, \text { loc }}^{p}(\Omega)$ we indicate the potential space

$$
\mathscr{L}_{2 s, \text { loc }}^{p}(\Omega):=\left\{u \in L^{p}(\Omega): u \eta \in \mathscr{L}_{2 s}^{p}\left(\mathbb{R}^{N}\right) \text { for any test function } \eta \in \mathcal{D}(\Omega)\right\} .
$$

Analogously, with $B_{p, 2, \text { loc }}^{2 s}(\Omega)$ we indicate the Besov space

$$
B_{p, 2, \text { loc }}^{2 s}(\Omega):=\left\{u \in L^{p}(\Omega): u \eta \in B_{p, 2}^{2 s}\left(\mathbb{R}^{N}\right) \text { for any test function } \eta \in \mathcal{D}(\Omega)\right\} .
$$

Moreover, our results guarantee that when the right hand side belongs to $L^{p}(\Omega \times(0, T))$ for $2 \leq p<\infty$ and for $1<p<2, s=1 / 2$, then the corresponding solution gains locally the maximum possible regularity, that is, it gains one time derivative and up to $2 s$ space derivatives, locally, in $L^{p}(\Omega)$. For $1<p<2$ and $s \neq 1 / 2$, instead, the local regularity is obtained in the Besov space $B_{p, 2, \text { loc }}^{2 s}(\Omega)$, which is strictly larger than $W_{\text {loc }}^{2 s, p}(\Omega)$. 
For the classical Laplace operator (which corresponds to the case $s=1$ ), this kind of results are standard, see e.g., [3, Theorem X.12], [7, Section 9], [10, Section 4.1]. Also, we recall [11, Theorem 1] for a more general result in an abstract setting.

Theorems 1.5 and 1.6 are natural extensions of analogous results of local regularity for the elliptic problem associated to the fractional Laplacian on a bounded domain, which have been obtained recently in [1, 2].

In the recent years, research on regularity of heat equations involving non-local terms has been very active. For instance, Hölder regularity was proved in [6, 9]. Boundary regularity has also been analyzed showing that, if $f=0$ and taking initial data $u(\cdot, 0)=u_{0} \in L^{2}(\Omega)$, the corresponding solution to (1.1) is such that $u(\cdot, t)$ belongs to $C^{s}\left(\mathbb{R}^{N}\right)$ for all $t>0$ and satisfies $u(\cdot, t) / \rho^{s} \in C^{s-\varepsilon}(\Omega)$ for any $\varepsilon>0$, $\rho(x)=\operatorname{dist}(x, \partial \Omega)$ being the distance to the boundary function. Concerning regularity in the Sobolev setting, we refer instead to [12, Theorem 26], where it has been proved the existence of a finite energy solution to (1.1), according to Definition 1.3 above. However, to the best of our knowledge, our Theorems 1.5 and 1.6 providing maximal space-time local regularity are new.

The controllability of parabolic equations involving non-local terms has also been investigated. We refer for instance to [5] where null controllability issues were addressed for heat equations involving nonlocal lower order terms. On the other hand, 13, 14 dealt with the control of heat equations involving the spectral fractional Laplacian (see 13, Section 1] for the definition of this operator), proving that null controllability holds for $s>1 / 2$, while for $s \leq 1 / 2$ the equation fails to be controllable. Notice that this operator does not coincide with (1.2).

The present paper is organized as follows. In Section 2, we will recall the sharp local regularity results obtained in [1, 2] for the elliptic problems associated to the fractional Laplacian. These results will be necessary in the proof of Theorems [1.5 and 1.6. In Section 3, we give the proof of Theorem [1.5, using the corresponding result for the classical Laplace operator in [4, Section 7.1.3, Theorem 5], employing a cut-off argument and using [1, Theorem 1.2]. In Section 4 we give the proof of Theorem [1.6 by applying

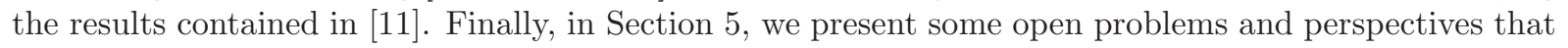
are closely related to our work.

\section{Regularity Results For the Elliptic PRoblem}

In this section, we recall some regularity results for weak solutions to the elliptic problem associated to the fractional Laplacian on a bounded open set. These results have been recently obtained in [1, 2], and they will be fundamental in the proof of Theorems [1.5] and 1.6. Therefore, throughout this section we are going to consider the following elliptic problem

$$
\begin{cases}(-\Delta)^{s} u=f & \text { in } \Omega, \\ u \equiv 0 & \text { on } \mathbb{R}^{N} \backslash \Omega .\end{cases}
$$

Let us start by recalling the definition of a weak solution, according to [1, 12.

Definition 2.1. Let $f \in W^{-s, 2}(\bar{\Omega})$. A function $u \in W_{0}^{s, 2}(\bar{\Omega})$ is said to be a finite energy solution to the Dirichlet problem (2.1) if for every $v \in W_{0}^{s, 2}(\bar{\Omega})$, the equality

$$
\frac{C_{N, s}}{2} \int_{\mathbb{R}^{N}} \int_{\mathbb{R}^{N}} \frac{(u(x)-u(y))(v(x)-v(y))}{|x-y|^{N+2 s}} d x d y=\langle f, v\rangle_{W^{-s, 2}(\bar{\Omega}), W_{0}^{s, 2}(\bar{\Omega})}
$$

holds.

We notice that, when $f \in L^{p}(\Omega)$ with $1<p<2$ and it does not belong to $W^{-s, 2}(\bar{\Omega})$, it is not natural to consider finite energy solutions for the problem (2.1). As for the parabolic problem above, we shall introduce an alternative notion of solution. This will be given by duality with respect to the following class of test functions:

$$
\mathcal{T}(\Omega)=\left\{\phi:(-\Delta)^{s} \phi=\psi \quad \text { in } \Omega, \phi=0 \quad \text { in } \mathbb{R}^{N} \backslash \Omega, \psi \in C_{0}^{\infty}(\Omega)\right\} .
$$


Definition 2.2. Let $f \in L^{1}(\Omega)$. We say that $u \in L^{1}(\Omega)$ is a weak duality or transposition solution to (2.1) if the equality

$$
\int_{\Omega} u \psi d x=\int_{\Omega} f \phi d x
$$

holds for any $\phi \in \mathcal{T}(\Omega)$ and $\psi \in C_{0}^{\infty}(\Omega)$.

The existence and uniqueness of finite energy weak solutions or the duality/transposition ones (depending on the regularity imposed on the right hand side term $f$ ) to problem (2.1) are guaranteed by [12, Theorem 12] and [12, Theorem 23], respectively. If $f \in L^{p}(\Omega)$, with $p \geq 2$, finite energy solutions of (2.1) will be considered while, if $1<p<2$, solutions will be understood in the sense of duality/transposition. In both cases, we shall refer to them as weak solutions. Moreover, we notice that, according to Definition 2.2. duality solutions do not require that $f$ belongs to the dual space $W^{-s, 2}(\bar{\Omega})$. Finally, we also notice that, if $f \in L^{p}(\Omega)$ with $p \geq 2$, we have the continuous embedding $L^{p}(\Omega) \hookrightarrow L^{2}(\Omega) \hookrightarrow W^{-s, 2}(\bar{\Omega})$, meaning that the property $f \in W^{-s, 2}(\bar{\Omega})$ is automatically guaranteed.

Concerning the regularity of the solutions to (2.1), the following result has been proved in [1, 2.

Theorem 2.3 ( $L^{p}$-Local elliptic regularity). Let $1<p<\infty$. Given $f \in L^{p}(\Omega)$, let $u$ be the unique weak solution to the Dirichlet problem (2.1). Then $u \in \mathscr{L}_{2 s, \text { loc }}^{p}(\Omega)$. As a consequence we have the following result.

(a) If $1<p<2$ and $s \neq 1 / 2$, then $u \in B_{p, 2, \text { loc }}^{2 s}(\Omega)$.

(b) If $1<p<2$ and $s=1 / 2$, then $u \in W_{\mathrm{loc}}^{2 s, p}(\Omega)=W_{\mathrm{loc}}^{1, p}(\Omega)$.

(c) If $2 \leq p<\infty$, then $u \in W_{\mathrm{loc}}^{2 s, p}(\Omega)$.

The proof of Theorem 2.3 requires a cut-off argument that allows us to reduce the problem to the whole space case, for which the result is already known. In particular, we have the following.

Theorem 2.4. Let $1<p<\infty$. Given $F \in L^{p}\left(\mathbb{R}^{N}\right)$, let $u$ be the unique weak solution to the fractional Poisson type equation

$$
(-\Delta)^{s} u=F \quad \text { in } \mathbb{R}^{N}
$$

Then $u \in \mathscr{L}_{2 s}^{p}\left(\mathbb{R}^{N}\right)$. As a consequence we have the following.

(a) If $1<p<2$ and $s \neq 1 / 2$, then $u \in B_{p, 2}^{2 s}\left(\mathbb{R}^{N}\right)$.

(b) If $1<p<2$ and $s=1 / 2$, then $u \in W^{2 s, p}\left(\mathbb{R}^{N}\right)=W^{1, p}\left(\mathbb{R}^{N}\right)$.

(c) If $2 \leq p<\infty$, then $u \in W^{2 s, p}\left(\mathbb{R}^{N}\right)$.

Theorem 2.4 is a classical result whose proof can be done by combining several results on singular integrals and Fourier transform contained in [16, Chapter V]. See also [1, 2]. In particular:

- If $1<p<2$ and $s \neq 1 / 2$, then the result follows from [16, Chapter V, Section 5.3, Theorem $5(\mathrm{~B})]$, which provides the inclusion $\mathscr{L}_{2 s}^{p}\left(\mathbb{R}^{N}\right) \subset B_{p, 2}^{2 s}\left(\mathbb{R}^{N}\right)$. Moreover, an explicit counterexample showing that sharper inclusions are not possible has been given in [16, Chapter V, Section 6.8].

- If $1<p<2$ and $s=1 / 2$, then applying [16, Chapter V, Section 3.3, Theorem 3] we have $\mathscr{L}_{2 s}^{p}\left(\mathbb{R}^{N}\right)=\mathscr{L}_{1}^{p}\left(\mathbb{R}^{N}\right)=W^{1, p}\left(\mathbb{R}^{N}\right)$.

- If $2 \leq p<\infty$, then [16, Chapter V, Section 5.3, Theorem 5(A)] yields $u \in B_{p, p}^{2 s}\left(\mathbb{R}^{N}\right)$ and this latter space, by definition, coincides with $W^{2 s, p}\left(\mathbb{R}^{N}\right)$ (see, e.g., [16. Chapter V, Section 5.1, Formula $(60)])$.

While developing the cut-off argument that we mentioned above, as an intermediate step we need to show that $u \in W^{s, p}(\Omega)$. Notice that, for $p \geq 2$, this is true for all weak solutions to (1.1) by classical embedding results. When $1<p<2$, instead, according to [12, Theorem 23], weak duality solutions to (2.1) are such that

$$
(-\Delta)^{\frac{s}{2}} u \in L^{p}(\Omega), \quad \forall p \in(1, N /(N-s))
$$

an this implies that $u \in W^{s, p}(\Omega)$ too. 
Proof of Theorem 2.3. For the sake of completeness we include the proof.

We start by noticing that, assuming $f \in L^{p}(\Omega), 1<p<\infty$, we have that (2.1) has a unique weak solution $u$ (either the finite-energy or the duality one) and that, from the discussion above, we have $u \in W^{s, p}(\Omega)$. In particular, $u \in L^{p}(\Omega)$.

As we have mentioned above, our strategy is based on a cut-off argument that will allow us to show that the solutions of the fractional Dirichlet problem in $\Omega$, after cut-off, are solutions of the elliptic problem on the whole space $\mathbb{R}^{N}$, for which Theorem 2.4 holds. For this purpose, given $\omega$ and $\widetilde{\omega}$ two open subsets of the domain $\Omega$ such that $\widetilde{\omega} \Subset \omega \Subset \Omega$, we introduce a cut-off function $\eta \in \mathcal{D}(\omega)$ such that

$$
\begin{cases}\eta(x) \equiv 1 & \text { if } x \in \widetilde{\omega} \\ 0 \leq \eta(x) \leq 1 & \text { if } x \in \omega \backslash \widetilde{\omega} \\ \eta(x)=0 & \text { if } x \in \mathbb{R}^{N} \backslash \omega .\end{cases}
$$

Let $\omega$ and $\eta \in \mathcal{D}(\omega)$ be respectively the set and the cut-off function constructed in (2.5). We consider the function $u \eta \in W^{s, p}\left(\mathbb{R}^{N}\right)$ and we have that $(-\Delta)^{s}(u \eta)$ is given by (see, e.g., [1, Proposition 1.5] or [15])

$$
(-\Delta)^{s}(u \eta)=\eta f+u(-\Delta)^{s} \eta-I_{s}(u, \eta)
$$

where $I_{s}(u, \eta)$ is a remainder term which is given by

$$
I_{s}(u, \eta)(x):=C_{N, s} \int_{\mathbb{R}^{N}} \frac{(u(x)-u(y))(\eta(x)-\eta(y))}{|x-y|^{N+2 s}} d y, \quad x \in \mathbb{R}^{N} .
$$

Let $\omega_{1}, \omega_{2}$ be open sets such that

$$
\bar{\omega} \subset \omega_{1} \subset \bar{\omega}_{1} \subset \omega_{2} \subset \bar{\omega}_{2} \subset \Omega .
$$

Since the function $\eta$ and the set $\omega$ in (2.5) are arbitrary, it follows that $u \in W^{s, p}\left(\omega_{2}\right)$. Thus we have $u \in W^{s, p}\left(\omega_{2}\right) \cap L^{p}(\Omega)$. Let

$$
g:=u(-\Delta)^{s} \eta-I_{s}(u, \eta)
$$

We now claim that $g \in L^{p}\left(\mathbb{R}^{N}\right)$ and there exists a constant $C>0$ such that

$$
\|g\|_{L^{p}\left(\mathbb{R}^{N}\right)} \leq C\left(\|u\|_{W^{s, p}\left(\omega_{2}\right)}+\|u\|_{L^{p}(\Omega)}\right) .
$$

Indeed, it is clear that $g$ is defined on all $\mathbb{R}^{N}$. Moreover

$$
\left\|u(-\Delta)^{s} \eta\right\|_{L^{p}\left(\mathbb{R}^{N}\right)}^{p}=\int_{\Omega}\left|u(-\Delta)^{s} \eta\right|^{p} d x \leq\left\|(-\Delta)^{s} \eta\right\|_{L^{\infty}(\Omega)}^{p}\|u\|_{L^{p}(\Omega)}^{p} .
$$

For estimating the term $I_{s}$, we use the decomposition

$$
\begin{aligned}
I_{s}(u, \eta)(x):= & C_{N, s} \int_{\mathbb{R}^{N}} \frac{(u(x)-u(y))(\eta(x)-\eta(y))}{|x-y|^{N+2 s}} d y \\
= & C_{N, s} \int_{\omega_{1}} \frac{(u(x)-u(y))(\eta(x)-\eta(y))}{|x-y|^{N+2 s}} d y \\
& +C_{N, s} \eta(x) \int_{\mathbb{R}^{N} \backslash \omega_{1}} \frac{u(x)-u(y)}{|x-y|^{N+2 s}} d y=\mathbb{I}_{1}(x)+\mathbb{I}_{2}(x), \quad x \in \mathbb{R}^{N},
\end{aligned}
$$

where we have set

$$
\mathbb{I}_{1}(x):=C_{N, s} \int_{\omega_{1}} \frac{(u(x)-u(y))(\eta(x)-\eta(y))}{|x-y|^{N+2 s}} d y, \quad x \in \mathbb{R}^{N},
$$

and

$$
\mathbb{I}_{2}(x):=C_{N, s} \eta(x) \int_{\mathbb{R}^{N} \backslash \omega_{1}} \frac{u(x)-u(y)}{|x-y|^{N+2 s}} d y, \quad x \in \mathbb{R}^{N} .
$$


Let $p^{\prime}:=p /(p-1)$. Using the Hölder inequality, we get that for a.e. $x \in \mathbb{R}^{N}$,

$$
\left|\mathbb{I}_{1}(x)\right| \leq C_{N, s}\left(\int_{\omega_{1}} \frac{|u(x)-u(y)|^{p}}{|x-y|^{N+s p}} d y\right)^{\frac{1}{p}}\left(\int_{\omega_{1}} \frac{|\eta(x)-\eta(y)|^{p^{\prime}}}{|x-y|^{N+s p^{\prime}}} d y\right)^{\frac{1}{p^{\prime}}} .
$$

Let $x \in \omega_{1}$ be fixed and $R>0$ such that $\omega_{1} \subset B(x, R)$. Using the Lipschitz continuity of the function $\eta$, we obtain that there exists constant $C>0$ such that

$$
\int_{\omega_{1}} \frac{|\eta(x)-\eta(y)|^{p^{\prime}}}{|x-y|^{N+s p^{\prime}}} d y \leq C \int_{\omega_{1}} \frac{d y}{|x-y|^{N+s p^{\prime}-p^{\prime}}} \leq C \int_{B(x, R)} \frac{d y}{|x-y|^{N+s p^{\prime}-p^{\prime}}} \leq C .
$$

In what follows, we will employ the following estimate. Let $A \subset \mathbb{R}^{N}$ be a bounded set and $B \subset \mathbb{R}^{N}$ an arbitrary set. Then there exists a constant $C>0$ (depending on $A$ and $B$ ) such that

$$
|x-y| \geq C(1+|y|), \quad \forall x \in A, \forall y \in \mathbb{R}^{N} \backslash B, \operatorname{dist}\left(A, \mathbb{R}^{N} \backslash B\right)=\delta>0 .
$$

Now, using (2.11), (2.12) and (2.13), we get

$$
\begin{aligned}
\int_{\mathbb{R}^{N}}\left|\mathbb{I}_{1}(x)\right|^{p} d x & \leq C\left(\int_{\omega_{2}} \int_{\omega_{1}} \frac{|u(x)-u(y)|^{p}}{|x-y|^{N+s p}} d y d x+\int_{\mathbb{R}^{N} \backslash \omega_{2}} \int_{\omega_{1}} \frac{|u(x)-u(y)|^{p}}{|x-y|^{N+s p}} d y d x\right) \\
& \leq C\left(\|u\|_{W^{s, p}\left(\omega_{2}\right)}^{p}+\int_{\mathbb{R}^{N} \backslash \omega_{2}} \int_{\omega_{1}} \frac{|u(x)|^{p}+|u(y)|^{p}}{(1+|x|)^{N+s p}} d y d x\right) \\
& \leq C\left(\|u\|_{W^{s, p}\left(\omega_{2}\right)}^{p}+\|u\|_{L^{p}(\Omega)}^{p}\right),
\end{aligned}
$$

where we have also used that $u=0$ on $\mathbb{R}^{N} \backslash \Omega$. Recall that $\mathbb{I}_{2}=0$ on $\mathbb{R}^{N} \backslash \omega$. Then using the Hölder inequality, we get that

$$
\left|\mathbb{I}_{2}(x)\right|^{p} \leq C\left(\int_{\mathbb{R}^{N} \backslash \omega_{1}} \frac{\eta^{p^{\prime}}(x) d y}{|x-y|^{N+s p^{\prime}}}\right)^{p-1} \int_{\mathbb{R}^{N} \backslash \omega_{1}} \frac{|u(x)-u(y)|^{p}}{|x-y|^{N+s p}} d y .
$$

For any $y \in \mathbb{R}^{N} \backslash \omega_{1}$, we have that

$$
\frac{\eta^{p^{\prime}}(x)}{|x-y|^{N+s p^{\prime}}}=\frac{\chi_{\bar{\omega}}(x) \eta^{p^{\prime}}(x)}{|x-y|^{N+s p^{\prime}}} \leq \chi_{\bar{\omega}}(x) \eta^{p^{\prime}}(x) \sup _{x \in \bar{\omega}} \frac{1}{|x-y|^{N+s p^{\prime}}} .
$$

So there exists a constant $C>0$ such that

$$
\int_{\mathbb{R}^{N} \backslash \omega_{1}} \frac{\eta^{p^{\prime}}(x) d y}{|x-y|^{N+s p^{\prime}}} \leq \chi_{\bar{\omega}}(x) \eta^{p^{\prime}}(x) \int_{\mathbb{R}^{N} \backslash \omega_{1}} \frac{d y}{\operatorname{dist}(y, \partial \bar{\omega})^{N+s p^{\prime}}} \leq C \chi_{\bar{\omega}}(x) \eta^{p^{\prime}}(x) .
$$

In (2.16) we have also used that the integral is finite which follows from the fact that $\operatorname{dist}\left(\partial \omega_{1}, \partial \bar{\omega}\right) \geq$ $\delta>0$ together with the fact that $\operatorname{dist}(y, \partial \bar{\omega})$ grows linearly as $y$ tends to infinity and $N+s p^{\prime}>N$.

Since $\chi_{\bar{\omega}} \eta^{p^{\prime}} \in L^{\infty}(\omega)$, and using (2.15), (2.16) and (2.13), we also get that there exists a constant $C>0$ such that

$$
\begin{aligned}
\int_{\mathbb{R}^{N}}\left|\mathbb{I}_{2}(x)\right|^{p} d x & =\int_{\omega}\left|\mathbb{I}_{2}(x)\right|^{p} d x \leq C \int_{\omega} \int_{\mathbb{R}^{N} \backslash \omega_{1}} \frac{|u(x)-u(y)|^{p}}{|x-y|^{N+s p}} d y d x \\
& \leq C \int_{\omega} \int_{\mathbb{R}^{N} \backslash \omega_{1}} \frac{|u(x)|^{p}+|u(y)|^{p}}{(1+|y|)^{N+s p}} d y d x \leq C\|u\|_{L^{p}(\Omega)}^{p},
\end{aligned}
$$

where we have used again that $u=0$ on $\mathbb{R}^{N} \backslash \Omega$. Estimate (2.9) follows from (2.10), (2.14), (2.17) and we have shown the claim. We therefore proved that $\eta u$ is a weak solution to the Poisson equation (2.3) with $F$ given by $F=\eta f+g$. Since $F \in L^{p}\left(\mathbb{R}^{N}\right)$, it follows from Theorem 2.4 that $\eta u \in \mathscr{L}_{2 s}^{p}\left(\mathbb{R}^{N}\right)$. We have shown that $u \in \mathscr{L}_{2 s \text {,loc }}^{p}(\Omega)$. As a consequence we have the following results.

(a) If $1<p<2$ and $s \neq 1 / 2$, then $\eta u \in B_{p, 2}^{2 s}\left(\mathbb{R}^{N}\right)$, hence $u \in B_{p, 2, \text { loc }}^{2 s}(\Omega)$.

(b) If $1<p<2$ and $s=1 / 2$, then $\eta u \in W^{2 s, p}\left(\mathbb{R}^{N}\right)=W^{1, p}\left(\mathbb{R}^{N}\right)$, hence $u \in W_{\text {loc }}^{2 s, p}(\Omega)=W_{\text {loc }}^{1, p}(\Omega)$. 
(c) If $2 \leq p<\infty$, then $\eta u \in W^{2 s, p}\left(\mathbb{R}^{N}\right)$, hence $u \in W_{\text {loc }}^{2 s, p}(\Omega)$.

The proof is finished.

We conclude this section mentioning that Theorem 2.3 can be proved also using techniques from pseudo-differential calculus (see, e.g., [8, Section 7] or [17, Chapter XI, Theorem 2.5]). Our approach is different and provides a proof based on basic estimates of solutions of general elliptic operators. In particular, our proofs do not require any knowledge of pseudo-differential operators theory.

\section{Proof of Theorem 1.5}

The proof of Theorem 1.5 employs a cut-off argument, as in Theorem 2.3 In particular:

- Firstly, we treat the case $\Omega=\mathbb{R}^{N}$, adapting the proof in [4, Section 7.1.3, Theorem 5] for the classical Laplace operator.

- The case of a general $\Omega$ is reduced to the previous one applying a cut-off argument.

3.1. The $W^{2 s, 2}$-regularity on $\mathbb{R}^{N}$. In this Section, we prove the $W^{2 s, 2}$-regularity result in the case where $\Omega$ is the whole space $\mathbb{R}^{N}$. We will adapt the proof presented in 4, Section 7.1.3, Theorem 5] for the local case.

Theorem 3.1. Assume $f \in L^{2}\left(\mathbb{R}^{N} \times(0, T)\right)$ and let $u \in L^{2}\left((0, T) ; W^{s, 2}\left(\mathbb{R}^{N}\right)\right) \cap C\left([0, T] ; L^{2}\left(\mathbb{R}^{N}\right)\right)$ with $u_{t} \in L^{2}\left((0, T) ; W^{-s, 2}\left(\mathbb{R}^{N}\right)\right)$ be the unique finite energy solution of the system

$$
\begin{cases}u_{t}+(-\Delta)^{s} u=f & \text { in } \mathbb{R}^{N} \times(0, T) \\ u(\cdot, 0) \equiv 0 & \text { on } \mathbb{R}^{N}\end{cases}
$$

Then

$$
u \in L^{2}\left((0, T) ; W^{2 s, 2}\left(\mathbb{R}^{N}\right)\right) \cap L^{\infty}\left((0, T) ; W^{s, 2}\left(\mathbb{R}^{N}\right)\right), \quad u_{t} \in L^{2}\left(\mathbb{R}^{N} \times(0, T)\right)
$$

Proof. First of all, we notice that the function $v:=u e^{-t}$ solves the system

$$
\begin{cases}v_{t}+(-\Delta)^{s} v+v=g & \text { in } \mathbb{R}^{N} \times[0, T], \\ v(\cdot, 0) \equiv 0 & \text { on } \mathbb{R}^{N},\end{cases}
$$

with $g:=f e^{-t} \in L^{2}\left(\mathbb{R}^{N} \times(0, T)\right)$. Now, multiplying (3.2) by $v_{t}$ and integrating by parts over $\mathbb{R}^{N}$ we obtain that

$$
\left(v_{t}, v_{t}\right)+B\left[v, v_{t}\right]+\left(v, v_{t}\right)=\left(g, v_{t}\right),
$$

where $(\cdot, \cdot)$ is the classical scalar product on $L^{2}\left(\mathbb{R}^{N}\right)$, while with $B[\cdot, \cdot]$ we indicated the bilinear form

$$
B[\phi, \psi]:=\frac{C_{N, s}}{2} \int_{\mathbb{R}^{N}} \int_{\mathbb{R}^{N}} \frac{(\phi(x)-\phi(y))(\psi(x)-\psi(y))}{|x-y|^{N+2 s}} d x d y .
$$

Moreover, we observe that

$$
B\left[v, v_{t}\right]=\frac{1}{2} \frac{d}{d t} B[v, v] \text { and }\left(v, v_{t}\right)=\frac{1}{2} \frac{d}{d t}(v, v) .
$$

Hence, using Young's inequality we have that, for every $\varepsilon>0$,

$$
\left\|v_{t}\right\|_{L^{2}\left(\mathbb{R}^{N}\right)}^{2}+\frac{1}{2} \frac{d}{d t}(B[v, v]+(v, v))=\left(g, v_{t}\right) \leq \frac{C}{\varepsilon}\|g\|_{L^{2}\left(\mathbb{R}^{N}\right)}^{2}+\varepsilon\left\|v_{t}\right\|_{L^{2}\left(\mathbb{R}^{N}\right)}^{2} .
$$

Choosing $\varepsilon \leq 1$ and integrating in time we find that

$$
\int_{0}^{T}\left\|v_{t}\right\|_{L^{2}\left(\mathbb{R}^{N}\right)}^{2} d t+\sup _{t \in[0, T]}(B[v(t), v(t)]+(v(t), v(t))) \leq C \int_{0}^{T}\|g\|_{L^{2}\left(\mathbb{R}^{N}\right)}^{2} d t,
$$

which implies that

$$
\left\|v_{t}\right\|_{L^{2}\left(\mathbb{R}^{N} \times(0, T)\right)}^{2}+\|v\|_{L^{\infty}\left((0, T), W^{s, 2}\left(\mathbb{R}^{N}\right)\right)} \leq C\|g\|_{L^{2}\left(\mathbb{R}^{N} \times(0, T)\right)}^{2} .
$$


Therefore,

$$
v \in L^{\infty}\left((0, T) ; W^{s, 2}\left(\mathbb{R}^{N}\right)\right), \quad v_{t} \in L^{2}\left(\mathbb{R}^{N} \times(0, T)\right)
$$

and, by definition, $u$ has the same regularity too. Finally, the $W^{2 s, 2}$ regularity for $u$ in the space variable is obtained in the following way. From (3.1) we have that $(-\Delta)^{s} u=f-u_{t} \in L^{2}\left(\mathbb{R}^{N} \times(0, T)\right)$. Hence, a. e. $t \in(0, T)$, we have that $(-\Delta)^{s} u(\cdot, t)=h(\cdot, t) \in L^{2}\left(\mathbb{R}^{N}\right)$ and, applying the regularity results for the elliptic case (see Theorem 2.4) we get that $u(\cdot, t) \in W^{2 s, 2}\left(\mathbb{R}^{N}\right)$ a. e. $t \in(0, T)$. Furthermore $u \in L^{2}\left((0, T) ; W^{2 s, 2}\left(\mathbb{R}^{N}\right)\right)$ and the proof is finished.

\subsection{The $W_{\text {loc }}^{2 s, 2}$-regularity in $\Omega$.}

Proof of Theorem 1.5. As we have mentioned above, our strategy is based on a cut-off argument that will allow us to show that solutions of the fractional parabolic problem in $\Omega$, after cut-off, are solutions of a problem on the whole space $\mathbb{R}^{N}$, for which Theorem 3.1 holds.

Let $f \in L^{2}(\Omega \times(0, T))$ and $u \in L^{2}\left((0, T) ; W_{0}^{s, 2}(\bar{\Omega})\right) \cap C\left([0, T] ; L^{2}(\Omega)\right)$ with $u_{t} \in L^{2}\left((0, T) ; W^{-s, 2}(\bar{\Omega})\right)$ be the unique finite energy solution to the system (1.1).

Let $\omega$ and $\eta \in \mathcal{D}(\omega)$ be respectively the set and the cut-off function constructed in (2.5). We consider the function $v:=u \eta$ and we write the equation satisfied by $v$. Recall from (2.6) that the fractional Laplacian of $v$ is given by

$$
(-\Delta)^{s} v=(-\Delta)^{s}(u \eta)=u(-\Delta)^{s} \eta+\eta(-\Delta)^{s} u-I_{s}(u, \eta)
$$

where the remainder term $I_{s}$ has been defined in (2.7). Then, $v$ is a solution to the following problem on $\mathbb{R}^{N}$ :

$$
\begin{cases}v_{t}+(-\Delta)^{s} v=F & \text { in } \mathbb{R}^{N} \times(0, T), \\ v(\cdot, 0) \equiv 0 & \text { on } \mathbb{R}^{N},\end{cases}
$$

with $F=\eta f+u(-\Delta)^{s} \eta-I_{s}(u, \eta)$.

Following the proof of [1] Theorem 1.2], we can show that $F \in L^{2}\left(\mathbb{R}^{N} \times(0, T)\right)$. Hence, from Theorem 3.1 we obtain that

$$
v \in L^{2}\left((0, T) ; W^{2 s, 2}\left(\mathbb{R}^{N}\right)\right) \cap L^{\infty}\left((0, T) ; W^{s, 2}\left(\mathbb{R}^{N}\right)\right), \quad v_{t} \in L^{2}\left(\mathbb{R}^{N} \times(0, T)\right) .
$$

This implies that $u \in L^{2}\left((0, T) ; W_{\text {loc }}^{2 s, 2}(\Omega)\right) \cap L^{\infty}\left((0, T) ; W_{0}^{s, 2}(\bar{\Omega})\right)$ and $u_{t} \in L^{2}(\Omega \times(0, T))$. The proof is finished.

\section{Proof of Theorem 1.6}

In this section, we prove the local regularity for the solutions to the parabolic problem (1.1), corresponding to a right hand side $f \in L^{p}(\Omega \times(0, T))$, with $1<p<\infty$.

First of all, notice that the following developments also apply to the case $p=2$. This special case has already been treated in the previous section, and there the proof of our local regularity Theorem 1.5 has been developed taking advantage of the Hilbert structure of the spaces $L^{2}(\Omega)$ and $L^{2}(\Omega \times(0, T))$.

Clearly that strategy cannot be extended to the general $L^{p}$ setting, and we have to adopt a different approach. This approach relies on an abstract result due to Lamberton [11. In particular, the proof of Theorem [1.6 will be a direct consequence of [11, Theorem 1]. For the sake of completeness, we recall its statement here.

Theorem 4.1. Let $(\Omega, \Sigma, m)$ be a measure space and let $A$ be the generator of a strongly continuous semigroup of linear operators $\left(\mathbb{T}_{t}\right)_{t \geq 0}$ on $L^{2}(\Omega, \Sigma, m)$ satisfying the following hypothesis:

(a) The semigroup $\left(\mathbb{T}_{t}\right)_{t \geq 0}$ is analytic and bounded on $L^{2}(\Omega, \Sigma, m)$.

(b) For every $p \in[1, \infty]$ and $\phi \in L^{p}(\Omega) \cap L^{2}(\Omega)$ we have the estimate

$$
\left\|\mathbb{T}_{t} \phi\right\|_{L^{p}(\Omega)} \leq\|\phi\|_{L^{p}(\Omega)}, \text { for all } t \geq 0 .
$$


Let $p \in(1, \infty)$. If $f \in L^{p}(\Omega \times(0, T))$, then the system

$$
\left\{\begin{array}{l}
u_{t}-A u=f, \quad t \in(0, T) \\
u(0)=0
\end{array}\right.
$$

admits a solution $u \in C\left([0, T] ; L^{p}(\Omega)\right)$, such that $u_{t}, A u \in L^{p}(\Omega \times(0, T))$.

Proof of Theorem 1.6. First of all notice that the operator $A=-(-\Delta)^{s}$ with domain

$$
\mathcal{D}(A)=\left\{u \in W_{0}^{s, 2}(\bar{\Omega}), \quad(-\Delta)^{s} u \in L^{2}(\Omega)\right\}
$$

is the generator of a submarkovian strongly continuous semigroup $\left(\mathbb{T}_{t}\right)_{t \geq 0}$ which is also ultracontractive (see, e.g., [1. Lemma 2.4]). Let $f \in L^{p}(\Omega \times(0, T))$ and let $u$ be the corresponding weak solution to the system (1.1). Then, it follows from Theorem 4.1 that $u_{t},(-\Delta)^{s} u \in L^{p}(\Omega \times(0, T))$. In particular we have that $(-\Delta)^{s} u(\cdot, t)=\left(f-u_{t}\right)(\cdot, t) \in L^{p}(\Omega)$ a. e. $t \in(0, T)$ and, according to Theorem 2.3 this implies that $u(\cdot, t) \in \mathscr{L}_{2 s, \text { loc }}^{p}(\Omega)$ a. e. $t \in(0, T)$. Therefore, for all $t \in(0, T)$ we have the following results.

(i) If $1<p<2$ and $s \neq 1 / 2$, then $u(\cdot, t) \in B_{p, 2, \text { loc }}^{2 s}(\Omega)$, a. e. $t \in(0, T)$.

(ii) If $1<p<2$ and $s=1 / 2$, then $u(\cdot, t) \in W_{\mathrm{loc}}^{2 s, p}(\Omega)=W_{\mathrm{loc}}^{1, p}(\Omega)$, a. e. $t \in(0, T)$.

(iii) If $2 \leq p<\infty$, then $u(\cdot, t) \in W_{\mathrm{loc}}^{2 s, p}(\Omega)$, a. e. $t \in(0, T)$.

Furthermore:

(a) If $1<p<2$ and $s \neq 1 / 2$, then $u \in L^{p}\left((0, T) ; B_{p, 2, \text { loc }}^{2 s}(\Omega)\right)$.

(b) If $1<p<2$ and $s=1 / 2$, then $u \in L^{p}\left((0, T) ; W_{\mathrm{loc}}^{2 s, p}(\Omega)\right)=L^{p}\left((0, T) ; W_{\mathrm{loc}}^{1, p}(\Omega)\right)$.

(c) If $2 \leq p<\infty$, then $u \in L^{p}\left((0, T) ; W_{\mathrm{loc}}^{2 s, p}(\Omega)\right)$.

The proof of the theorem is finished.

We conclude this section with the following remark.

Remark 4.2. Recall that we have said in the proof of Theorem 1.6 that the operator $A=-(-\Delta)^{s}$ with domain given by (4.1) generates a strongly continuous submarkovian semigroup $\left(\mathbb{T}_{t}\right)_{t \geq 0}$ on $L^{2}(\Omega)$ and the semigroup is analytic and ultracontractive. This implies that the semigroup can be extended to contraction semigroups on $L^{p}(\Omega)$ for all $p \in[1, \infty]$ and each semigroup is strongly continuous if $p \in[1, \infty)$ and bounded analytic if $p \in(1, \infty)$. Let $A_{p}$ denote the generator of the semigroup on $L^{p}(\Omega)$ for $p \in[1, \infty]$ so that $A_{2}$ coincides with $A$. By Theorem 4.1 if $1<p<\infty$ and $f \in L^{p}(\Omega \times(0, T))$, then the unique solution $u \in C\left([0, T] ; L^{p}(\Omega)\right)$ of the system (1.1) has the following regularity:

$$
u \in L^{p}\left((0, T) ; D\left(A_{p}\right)\right) .
$$

This trivially implies that $A_{p} u \in L^{p}(\Omega \times(0, T))$ and $u_{t} \in L^{p}(\Omega \times(0, T))$. Our contribution in the present paper was to show that $D\left(A_{p}\right) \subset \mathscr{L}_{2 s, \text { loc }}^{p}(\Omega)$ for every $1<p<\infty$.

\section{OPEN PROBLEMS AND PERSPECTIVES}

In the present paper we proved that weak solutions to the parabolic problem for the fractional Laplacian, with a non-homogeneous right-hand side $f \in L^{p}(\Omega \times(0, T))(1<p<\infty)$ and zero initial datum, belong to $L^{p}\left((0, T) ; \mathscr{L}_{2 s, \text { loc }}^{p}(\Omega)\right)$. The following comments are worth considering.

(a) A natural interesting extension of our result would be the analysis of the global maximal regularity in space for weak solutions to (1.1). The problem is delicate however.

Indeed, already at the elliptic level, we know that even if $\Omega$ has a smooth boundary, then the global maximal regularity up to the boundary does not hold. To be more precise, assume that $\Omega$ has a smooth boundary, $f \in L^{p}(\Omega)(1<p<\infty)$ and let $u$ be the associated weak solution to the Dirichlet problem (2.1). It is known that, if $p \geq 2$, then $u$ does not always belongs to $W^{2 s, p}(\Omega)$ and, if $1<p<2$, then $u$ does not always belong to $B_{p, 2}^{2 s}(\Omega)$. This shows that in general, the corresponding weak solution $u$ to the parabolic system (1.1) does not always belong to $L^{p}\left((0, T) ; W^{2 s, p}(\Omega)\right)$ if $p \geq 2$ and does not always belong to $L^{p}\left((0, T) ; B_{p, 2}^{2 s}(\Omega)\right)$ if $1<p<2$. 
On the one hand, Theorem 4.1 shows that $u \in L^{p}\left((0, T) ; D\left(A_{p}\right)\right)$, that is, in particular $u(\cdot, t) \in$ $D\left(A_{p}\right)$ for a.e. $t \in(0, T)$. On the other hand, according to the discussions given in [1, Section $5]$, at least if $\Omega$ has a sufficiently smooth boundary, one has that $u(\cdot, t)=\rho^{s} v(\cdot, t)$ where $v(\cdot, t)$ is a regular function up to the boundary. Here, $\rho(x):=\operatorname{dist}(x, \partial \Omega)$ for $x \in \Omega$. In addition one could expect that $\rho^{-s} u, \rho^{1-s} u \in L^{p}\left((0, T) ; L^{p}\left((0, T) ; W^{2 s, p}(\Omega)\right)\right.$ if $2 \leq p<\infty$, and $\rho^{-s} u, \rho^{1-s} u \in$ $L^{p}\left((0, T) ; L^{p}\left((0, T) ; B_{p, 2}^{2 s}(\Omega)\right)\right.$ if $1<p<2$. This constitutes an interesting open problem. We refer to [1, Section 5] for a more complete discussion on related topics and the difficulties that it raises.

(b) It would be interesting to consider the case of a non-zero initial datum in equation (1.1). In the Hilbert space framework, i.e. when working in the $L^{2}(\Omega)$ setting, the strategy of Section 3 can be extended to deal with initial data in $W_{0}^{s, 2}(\bar{\Omega})$. To the best of our knowledge, the corresponding analogous result in the case $p \in(1, \infty), p \neq 2$, is still unknown.

\section{REFERENCES}

[1] U. Biccari, M. Warma and E. Zuazua. Local elliptic regularity for the Dirichlet fractional Laplacian. Adv. Nonlinear Stud. 17 (2017), 387-409.

[2] U. Biccari, M. Warma and E. Zuazua. Addendum: Local elliptic regularity for the Dirichlet fractional Laplacian.

[3] H. Brezis. Functional Analysis, Sobolev Spaces and Partial Differential Equations. Springer Science \& Business Media, 2010.

[4] L.C. Evans. Partial Differential Equations. American Mathematical Society, 2010.

[5] E. Fernández-Cara, Q. Lü and E. Zuazua. Null controllability of linear heat and wave equations with nonlocal in space terms. SIAM J. Control Optim. 54 (2016), 2009-2019.

[6] X. Fernández-Real and X. Ros-Oton. Boundary regularity for the fractional heat equation. Rev. R. Acad. Cienc. Exactas Fís. Nat. Ser. A Math. RACSAM 110 (2016), 49-64.

[7] P. Grisvard. Équations différentielles abstraites. Ann. Sci. École Norm. Sup. 2 (1969), 311-395.

[8] G. Grubb, Fractional Laplacians on domains, a development of Hörmanders theory of $\mu$-transmission pseudodifferential operators. Adv. Math. 268 (2015), 478-528.

[9] M. Kassmann and R.W. Schwab. Regularity results for nonlocal parabolic equations. Riv. Mat. Univ. Parma., (1) 5 (2014), 183-212.

[10] O.A. Ladyzhenskaya, V.A. Solonnikov and N.N. Uraltseva. Linear and Quasi-linear Equations of Parabolic Type. American Mathematical Society, 1968.

[11] D. Lamberton. Equations d'évolution linéaires associées à des semi-groupes de contractions dans les espaces $L^{p}$. J. Funct. Anal. 72 (1987), 252-262.

[12] T. Leonori, I. Peral, A. Primo and F. Soria. Basic estimates for solutions of a class of nonlocal elliptic and parabolic equations. Discrete Contin. Dyn. Syst. 35 (2015), 6031-6068.

[13] S. Micu and E. Zuazua. On the controllability of a fractional order parabolic equation. SIAM J. Control Optim. 44 (2006), 1950-1972.

[14] L. Miller. On the controllability of anomalous diffusions generated by the fractional Laplacian. Math. Control Signals Systems. 18 (2006), 260-271.

[15] X. Ros-Oton and J. Serra. The Dirichlet problem for the fractional Laplacian: regularity up to the boundary. J. Math. Pures Appl. 101 (2014), 275-302.

[16] E. Stein Singular Integrals and Differentiability Properties of Functions. Princeton Mathematical Series, 1970.

[17] M.E. Taylor. Pseudodifferential operators. Princeton Mathematical Series, Vol. 4, 1981.

[18] H. Triebel. Theory of Function Spaces II. Monographs in Mathematics, 84. Birkhäuser Verlag, Basel, Boston, Berlin, 1992.

Umberto Biccari, Deustotech, University of Deusto, 48007 Bilbao, Basque Country, Spain.

Umberto Biccari, Facultad de Ingeniería, Universidad de Deusto, Avda Universidades 24, 48007 Bilbao, BAsque Country, Spain.

E-mail address: umberto.biccari@deusto.es, u.biccari@gmail.com

Mahamadi Warma, University of Puerto Rico (Rio Piedras Campus), College of Natural Sciences, Department of Mathematics, PO Box 70377 San Juan PR 00936-8377 (USA).

E-mail address: mahamadi.warma1@upr.edu, mjwarma@gmail.com

Enrique Zuazua, Deustotech, University of Deusto, 48007 Bilbao, Basque Country, Spain.

Enrique Zuazua, Facultad de Ingeniería, Universidad de Deusto, Avda Universidades 24, 48007 Bilbao, Basque Country, SPAin. 
Enrique Zuazua, Departamento de Matemáticas, Universidad Autónoma de Madrid, Campus de Cantoblanco, 28049, MAdRID, SPAIN

E-mail address: enrique.zuazua@deusto.es, enrique.zuazua@uam.es 